\title{
A uniqueness result for one-dimensional inverse scattering
}

\author{
C. Bennewitz*1, B. M. Brown ${ }^{* * 2}$, and R. Weikard ${ }^{* * * 3}$ \\ ${ }^{1}$ Department of Mathematics, Lund University, Box 118, SE-221 00 Lund, Sweden \\ ${ }^{2}$ Cardiff School of Computer Science \& Informatics, Cardiff University, Queen's Buildings, 5 The Parade, \\ Roath, Cardiff CF24 3AA, UK \\ ${ }^{3}$ Department of Mathematics, University of Alabama at Birmingham, Birmingham, AL 35226-1170, USA
}

Received 12 April 2011, revised 21 June 2011, accepted 23 June 2011

Published online 9 February 2012

Key words Inverse scattering, $m$-function, one-dimensional problems, left and right definite problems MSC (2010) 34K29

We consider the whole-line inverse scattering problem for Sturm-Liouville equations which have constant coefficients on a half-line. Since in this case the reflection coefficient determines a Weyl-Titchmarsh $m$-function, it determines the coefficients up to some simple Liouville transformations. Given inverse spectral theory, proofs are fairly simple but provide extensions of known results as we require less smoothness and less decay than is customary.

(C) 2012 WILEY-VCH Verlag GmbH \& Co. KGaA, Weinheim

\section{Introduction}

Quantum-mechanical inverse scattering, leading to problems posed on a half-line because of the assumed spherical symmetry, were first investigated by the physicists Fröberg [13] and Hylleraas [17] introducing the scattering phase as data. Levinson [18] proved uniqueness if there are no eigenvalues, and Marchenko [20] showed uniqueness under the assumption that the potential has a finite first moment. This condition guarantees that there are only finitely many eigenvalues.

The first results for whole-line scattering were obtained by Faddeev [12] in 1964. More details and further references can be found in Deift and Trubowitz [10], Levitan [19] and Marchenko [21].

It has long been apparent that there is a close connection between one-dimensional inverse spectral theory and inverse scattering, but it has never been made clear exactly what the connection is in the whole line case. In this note we will use a very simple connection in the case when the equation has constant coefficients on a half-axis. This will yield a uniqueness theorem for inverse scattering under this special assumption, but which is in other respects considerably more general than standard results. Our Theorem 5.2 makes a statement about meromorphic extensions of reflection coefficients and also gives a formula connecting such a meromorphic extension with a half-line Titchmarsh-Weyl $m$-coefficient. Neither of these are new. The existence of a meromorphic extension of the reflection coefficient in certain circumstances has been noted by many authors, among them Aktosun, Klaus, and van der Mee [1], Grebert and Weder [15], and Deift and Killip [9]. Recently a very thorough investigation of this question was carried out by Egorova and Teschl [11]. The formula connecting reflection coefficient and $m$-coefficient seems not to be so well-known, but can be found in Gesztesy and Simon [14] — under significantly more general circumstances—and also in Deift and Killip [9]. Since uniqueness results for inverse spectral theory are valid for far more general equations than those considered in standard inverse scattering theory we thought it worthwhile to point out the connection again, in particular, since we were able to extend it to new circumstances. Our main result is thus Theorem 5.3.

\footnotetext{
* e-mail: christer.bennewitz@math.lu.se, Phone: +4646222 8563, Fax: +46222 4213

** e-mail: malcolm@cs.cf.ac.uk, Phone: +44 (0)292087 4812, Fax: +44 (0)292087 4598

*** Corresponding author: e-mail: rudi@math.uab.edu, Phone: +1 205 9342154, Fax: +1 2059349025
} 
In particular we do not need the usual assumption of a finite first moment for the potential in the case of the one-dimensional Schrödinger equation, we will be able to deal with acoustical scattering without the usual smoothness assumptions, and we will also consider left definite problems. The last point is of importance in applying the scattering/inverse scattering method for studying the Camassa-Holm equation, an integrable system which has aroused a great deal of interest in recent times (see e.g., Constantin [8]).

Since we do not need a finite first moment for the potential we can deal with cases where there are infinitely many eigenvalues accumulating at the beginning of the continuous spectrum. We can also deal with situations where eigenvalues accumulate at $-\infty$ or both at $-\infty$ and the beginning of the continuous spectrum.

\section{The equation}

Consider the general Sturm-Liouville equation

$$
-\left(p f^{\prime}\right)^{\prime}+q f=\lambda w f
$$

on a real interval $I$, with the general assumptions that $1 / p, q$ and $w$ are real-valued and locally integrable. If $w \geq 0$ a suitable Hilbert space for studying this equation is the weighted $L^{2}$-space with norm-square

$$
\|f\|^{2}=\int_{I}|f|^{2} w
$$

and it is well-known that it is possible to define at least one selfadjoint operator in this space associated with Equation (2.1). Assuming that $w>0$ a.e. in $I$ the change of variable $t=\int_{a}^{x} w$, where $a \in I$, will transform the equation to one for which $w \equiv 1$. This change of variable gives a unitary map between the corresponding Hilbert spaces.

If $w$ is not of fixed sign but instead $p$ and $q$ are non-negative and $q$ is not zero a.e. one may instead consider (2.1) in a Hilbert space with norm-square

$$
\|f\|^{2}=\int_{I}\left(p\left|f^{\prime}\right|^{2}+q|f|^{2}\right)
$$

and develop a spectral theory for (2.1) in this space (see [2], [3], [6] and others). This case is often referred to as the left definite case in contrast to the more standard right definite case where $w \geq 0$. In the left definite case the change of variable $t=\int_{a}^{x} 1 / p$ transforms (2.1) into an equation with $p \equiv 1$.

We will therefore consider two cases, namely the right definite equation

$$
-\left(p f^{\prime}\right)^{\prime}+q f=\lambda f
$$

and the left definite equation

$$
-f^{\prime \prime}+q f=\lambda w f
$$

where $q$ is non-negative and does not vanish a.e. Note that in the right definite case we do not assume that $p$ has a fixed sign. Similarly, in the left definite case we do not assume that $w$ has a fixed sign.

\section{Jost solutions and scattering data}

Consider Equation (2.1) on $\mathbb{R}$, where $1 / p, q$ and $w$ are real-valued and locally integrable. In order to discuss scattering this equation needs to be close, in some sense, to a constant coefficient equation, say $-f^{\prime \prime}+q_{0} f=\lambda f$. We therefore also assume that $1-1 / p, q-q_{0}$ and $w-1$ are in $L^{1}(\mathbb{R})$. This implies the existence of Jost solutions $f_{ \pm}$.

Theorem 3.1 Suppose there is a constant $q_{0}$ such that $q-q_{0}, 1-1 / p$ and $w-1$ are all in $L^{1}(\mathbb{R})$ and let $\lambda=q_{0}+k^{2}$ where $\operatorname{Im} k \geq 0, k \neq 0$. Then there exist solutions $f_{ \pm}(\cdot, k)$ of $(2.1)$ such that

- $f_{+}(x, k) \sim e^{i k x}$ and $p f_{+}^{\prime}(x, k) \sim i k e^{i k x}$ as $x \rightarrow \infty$,

- $f_{-}(x, k) \sim e^{-i k x}$ and $p f_{-}^{\prime}(x, k) \sim-i k e^{-i k x}$ as $x \rightarrow-\infty$.

These so-called Jost solutions are, locally in $x$, continuous in $\operatorname{Im} k \geq 0, k \neq 0$ and analytic in $\operatorname{Im} k>0$. 
Proof. We prove the existence of $f_{+}$; the existence of $f_{-}$is proved similarly.

Setting $F=\left(\begin{array}{c}f \\ p f^{\prime}\end{array}\right)$ Equation (2.1) is equivalent to the first order system

$$
F^{\prime}=\left(\begin{array}{cc}
0 & 1 / p \\
q-\lambda w & 0
\end{array}\right) F
$$

We are looking for $F$ which is asymptotic to $e^{i k x}\left(\begin{array}{c}1 \\ i k\end{array}\right)$ as $x \rightarrow \infty$ so it is natural to define

$$
G(x, k)=e^{-i k x}\left(\begin{array}{cc}
1 & 0 \\
0 & 1 / i k
\end{array}\right) F(x, k) .
$$

It is then easily verified that $F$ will have the desired properties if $G$ is a solution of the integral equation

$$
G(x, k)=\left(\begin{array}{l}
1 \\
1
\end{array}\right)+i k \int_{x}^{\infty} M(\cdot, x, k)\left(\begin{array}{cc}
u & 0 \\
0 & 1-1 / p
\end{array}\right) G(\cdot, k)
$$

where $u(\cdot, k)=1-w+\left(q-q_{0} w\right) / k^{2}$ and

$$
M(t, x, k)=\frac{1}{2}\left(\begin{array}{ll}
1-e^{2 i k(t-x)} & 1+e^{2 i k(t-x)} \\
1+e^{2 i k(t-x)} & 1-e^{2 i k(t-x)}
\end{array}\right) .
$$

Since $\operatorname{Im} k \geq 0$ and $t \geq x$ the matrix norm of $M$ is easily seen to be 1 , and the matrix norm of $\left(\begin{array}{cc}u & 0 \\ 0 & 1-1 / p\end{array}\right)$ is $\max (|u|,|1-1 / p|)$, which by assumption is integrable. Thus successive approximations will lead to a bounded solution $G$ of (3.1) with the desired continuity and analyticity properties and satisfying the estimate

$$
\|G(x, k)\| \leq \sqrt{2} \exp \left(|k| \int_{x}^{\infty} \max (|u|,|1-1 / p|)\right) .
$$

Define the Wronskian $\left[f_{1}, f_{2}\right]$ of two solutions of (2.1) by

$$
\left[f_{1}, f_{2}\right]=f_{1} p f_{2}^{\prime}-f_{2} p f_{1}^{\prime} .
$$

Differentiation and use of the equation shows that the Wronskian of two solutions is constant, and it vanishes precisely if the solutions are linearly dependent.

For real $k \neq 0$ the functions $f_{ \pm}(\cdot,-k)$ satisfy the same equation as $f_{ \pm}(\cdot, k)$. Because of the asymptotics of Theorem 3.1 it is clear that

$$
\left[f_{+}(\cdot,-k), f_{+}(\cdot, k)\right]=\left[f_{-}(\cdot, k), f_{-}(\cdot,-k)\right]=2 i k,
$$

so that $f_{+}(\cdot, k)$ and $f_{+}(\cdot,-k)=\overline{f_{+}(\cdot, k)}$, as well as the functions $f_{-}(\cdot, k)$ and $f_{-}(\cdot,-k)=\overline{f_{-}(\cdot, k)}$, are bases of the solution-space for $\lambda=q_{0}+k^{2}, k \in \mathbb{R} \backslash\{0\}$. We therefore have

$$
\begin{aligned}
& f_{-}(\cdot, k)=a f_{+}(\cdot, k)+b f_{+}(\cdot,-k), \\
& f_{-}(\cdot,-k)=\bar{a} f_{+}(\cdot,-k)+\bar{b} f_{+}(\cdot, k) .
\end{aligned}
$$

Taking Wronskians this gives

$$
\left[f_{-}(\cdot, k), f_{-}(\cdot,-k)\right]=\left(|a|^{2}-|b|^{2}\right)\left[f_{+}(\cdot, k), f_{+}(\cdot,-k)\right]
$$

so that $1+|a|^{2}=|b|^{2}$. Thus $b \neq 0$ and setting $\mathfrak{T}_{+}=1 / b, \mathfrak{R}_{+}=a / b$ we obtain $\left|\mathfrak{T}_{+}\right|^{2}+\left|\mathfrak{R}_{+}\right|^{2}=1$ and

$$
\mathfrak{T}_{+}(k) f_{-}(\cdot, k)=\mathfrak{R}_{+}(k) f_{+}(\cdot, k)+f_{+}(\cdot,-k) .
$$

Similarly we obtain

$$
\mathfrak{T}_{-}(k) f_{+}(\cdot, k)=\mathfrak{R}_{-}(k) f_{-}(\cdot, k)+f_{-}(\cdot,-k) .
$$


Taking Wronskians of these equations with $f_{+}(\cdot, \pm k)$ and $f_{-}(\cdot, \pm k)$ respectively shows that $\mathfrak{T}_{+}=\mathfrak{T}_{-}$, so that we drop the subscript from now on, and

$$
\begin{aligned}
& \mathfrak{T}(k)=2 i k /\left[f_{-}(\cdot, k), f_{+}(\cdot, k)\right], \\
& \mathfrak{R}_{+}(k) / \mathfrak{T}(k)=-\overline{\mathfrak{R}_{-}(k) / \mathfrak{T}(k)}=\left[f_{+}(\cdot,-k), f_{-}(\cdot, k)\right] / 2 i k .
\end{aligned}
$$

$\mathfrak{T}$ is the transmission coefficient and $\mathfrak{R}_{ \pm}$the reflection coefficients for our equation, and the scattering matrix $S(k)=\left(\begin{array}{cc}\mathfrak{T}(k) & \mathfrak{R}_{+}(k) \\ \mathfrak{R}_{-}(k) & \mathfrak{T}(k)\end{array}\right)$ is unitary and a continuous function of $k$ for real $k \neq 0$. This is a primary datum in a scattering experiment.

Since $f_{ \pm}(\cdot, k)$ is analytic in the upper half plane (3.3) gives a meromorphic continuation of $\mathfrak{T}$ to the upper half plane. In general $\mathfrak{R}_{ \pm}$do not have such extensions.

The poles of $\mathfrak{T}$ are found exactly where $f_{+}$and $f_{-}$are linearly dependent. As we have seen this cannot happen for real $k \neq 0$. If $\operatorname{Im} k>0$, then $f_{+}$decays exponentially for large and $f_{-}$for small $x$, so if $f_{+}$and $f_{-}$are linearly dependent they decay exponentially at $\pm \infty$.

We shall call $\lambda=q_{0}+k^{2}$ an eigenvalue if $k$ is a pole of $\mathfrak{T}$. If $\lambda$ is an eigenvalue an integration by parts shows that

$$
\int_{-\infty}^{\infty}\left(p\left|f_{+}^{\prime}\right|^{2}+q\left|f_{+}\right|^{2}\right)=\lambda \int_{-\infty}^{\infty}\left|f_{+}\right|^{2} w
$$

since the integrated terms vanish at $\pm \infty$ by exponential decay, which also implies that the integrals converge. The left integral cannot be zero in the left definite case, and the right integral cannot be zero in the right definite case, and this implies that eigenvalues must be real, so that the corresponding $k$ is purely imaginary. Thus all poles of $\mathfrak{T}$ are located on the positive imaginary $k$-axis.

Note that neither in the right definite, nor in the left definite case does the function $f_{+}(\cdot, k)$ for real $k \neq 0$ have finite norm over an interval $[a, \infty)$. In the right definite case it is well-known that this implies that there is no $\lambda \in \mathbb{C}$ for which all solutions of (2.2) have finite norm over $[a, \infty)$ (see [16, Theorem 10.1.2]). A similar statement is true in the left definite case (see [5]). In particular, if $\operatorname{Im} k>0$ the only solutions with finite norm are multiples of $f_{+}(\cdot, k)$.

\section{Titchmarsh-Weyl theory}

Consider the right or left definite equation (2.1) under the same assumptions on the coefficients as in Theorem 3.1, but on an interval $[a, \infty)$. As noted at the end of the previous section, if $\operatorname{Im} k>0$ the only solutions of (2.1) with finite norm are then multiples of $f_{+}(\cdot, k)$ (the "limit point" case).

Consider the right definite case. Then if $\operatorname{Im} \lambda \neq 0$ there is a unique solution (the Weyl solution) $\psi(\cdot, \lambda)$ of $(2.2)$ in $L^{2}(a, \infty)$ with $\psi(a, \lambda)=1$. We define the Dirichlet $m$-coefficient by

$$
m(\lambda)=p \psi^{\prime}(a, \lambda) .
$$

It is clear we also have $m(\lambda)=p f_{+}^{\prime}(a, k) / f_{+}(a, k)$ where $\lambda=k^{2} \notin \mathbb{R}$.

The importance of the $m$-coefficient in spectral theory is that it contains all the spectral information of a corresponding selfadjoint operator. For our purposes, however, the central fact is the following theorem and its corollaries.

We consider, in addition to Equation (2.2) on $[a, \infty)$ a similar equation

$$
-\left(\breve{p} f^{\prime}\right)^{\prime}+\breve{q} f=\lambda f,
$$

also on $[a, \infty)$. Define $h(x)=\int_{a}^{x}|p|^{-1 / 2}, \breve{h}(x)=\int_{a}^{x}|\breve{p}|^{-1 / 2}, s=\breve{h}^{-1} \circ h$ and $r=\sqrt{s^{\prime}}$. We then have the following theorem.

Theorem 4.1 Suppose (2.2) and (4.1) have the same Dirichlet $m$-coefficient for $[a, \infty)$. Then $s, r$ and $p r^{\prime}$ are locally absolutely continuous, $r(a)=1, p r^{\prime}(a)=0$ and

$$
\breve{p} \circ s=r^{4} p, \quad \breve{q} \circ s=\left(-\left(p r^{\prime}\right)^{\prime}+q r\right) / r .
$$

Conversely, if there is such a relation between the coefficients, then the two equations have the same m-coefficient. 
For the proof we refer to [4], especially Theorem 4.2.

Thus the $m$-coefficient determines the equation up to a simple type of transformation of the coefficients. With additional assumptions the equation is uniquely determined by the $m$-coefficient. We give two corollaries of this kind.

Corollary 4.2 Under the assumptions of Theorem 4.1 , if in addition $|\breve{p}|=|p|$ in $[a, \infty)$, then $\breve{p}=p$ and $\breve{q}=q$. Proof. The extra assumption implies that $s(x)=x, r \equiv 1$.

Corollary 4.3 Under the assumptions of Theorem 4.1 , if $\breve{q}=q$ is constant, then $\breve{p}=p$.

Proof. The extra assumption means that $\left(p r^{\prime}\right)^{\prime}=0$ so that $p r^{\prime}$ is constant. But $p r^{\prime}(a)=0$, so that $p r^{\prime} \equiv 0$. Thus $r$ is constant. Since $r(a)=1$ we obtain $r \equiv 1$, so that $s^{\prime}=1$. Since $s(a)=a$ it follows that $s(x)=x$.

Very similar results are true for a left definite equation. More specifically, in this case there exists a unique solution $\psi$ of (2.3) with finite norm over $[a, \infty)$ for which $\psi(a, \lambda)=1 / \lambda$ if $\operatorname{Im} \lambda \neq 0$, and we define the left definite Dirichlet $m$-coefficient by $m(\lambda)=\psi^{\prime}(a, \lambda)$. Again, clearly $m(\lambda)=f_{+}^{\prime}(a, k) /\left(\lambda f_{+}(a, k)\right)$ where $\lambda=q_{0}+k^{2} \notin \mathbb{R}$.

Now consider, in addition to (2.3) on $[a, \infty)$, another left definite equation

$$
-f^{\prime \prime}+\breve{q} f=\lambda \breve{w} f
$$

on the same interval. We now define $h(x)=\int_{a}^{x} \sqrt{|w|}, \breve{h}(x)=\int_{a}^{x} \sqrt{|\breve{w}|}, s=\breve{h}^{-1} \circ h$ and $r=1 / \sqrt{s^{\prime}}$. We then have the following theorem.

Theorem 4.4 Suppose that in the left definite equations (2.3) and (4.2) $\operatorname{supp} w=\operatorname{supp} \breve{w}=\mathbb{R}$, and that they have the same Dirichlet $m$-coefficient for $[a, \infty)$. Then $s, r$ and $r^{\prime}$ are locally absolutely continuous, $r(a)=1$, $r^{\prime}(a)=0$ and

$$
\breve{w} \circ s=r^{4} w, \quad \breve{q} \circ s=r^{3}\left(-r^{\prime \prime}+q r\right) .
$$

Conversely, if there is such a relation between the coefficients, then the two equations have the same m-coefficient.

For the proof we refer to [6], especially Theorem 4.4.

We again give two corollaries which show that with additional assumptions the $m$-coefficient determines the equation uniquely.

Corollary 4.5 Under the assumptions of Theorem 4.4 , if in addition $|\breve{w}|=|w|$ in $[a, \infty)$, then $\breve{w}=w$ and $\breve{q}=q$.

Proof. The assumption implies that $s(x)=x$ so that $r \equiv 1$.

Corollary 4.6 Under the assumptions of Theorem 4.4 , if $\breve{q}=q$, then $\breve{w}=w$.

For the proof we refer to [6], especially Corollary 4.6.

\section{Inverse scattering}

The uniqueness problem of inverse scattering is to determine to which extent the equation is determined by the scattering matrix $S$. The standard result for the one-dimensional Schrödinger equation $((2.2)$ with $p \equiv 1)$ is that knowledge of $\mathfrak{R}_{+}$(or $\mathfrak{R}_{-}$) together with the location of any eigenvalues and the values of corresponding "normalisation constants" determine $q$, provided it is assumed that the first moment $\int_{\mathbb{R}}|t q(t)| d t$ of $q$ is finite. This condition implies that there are only finitely many eigenvalues (see [10, Lemma 2.2]).

We shall make use of the following well-known lemma.

Lemma 5.1 Suppose that $v$ is continuous on some open subinterval I of $\mathbb{R}$. Then $v$ has at most one meromorphic extension to the upper half plane.

The proof depends on the fact that the difference of two extensions is meromorphic in the upper half plane with zero boundary values on $I$. By the reflection principle it therefore extends analytically to a neighbourhood of $I$ while it vanishes on $I$ and is therefore identically zero.

The connection between spectral and scattering theory is given by the following simple theorem (the possibility of a meromorphic extension of $\mathfrak{R}_{-}$has been noted by Aktosun, Klaus, and van der Mee [1], by Grebert and Weder [15] by Egorova and Teschl [11], and and others while the connection between $m$ and $\Re_{-}$has been used at least by Gesztesy and Simon [14] and Deift and Killip [9]). 
Theorem 5.2 Suppose that in Equation (2.1) we have $1-1 / p, w-1$ and $q-q_{0}$ in $L^{1}(\mathbb{R})$, where $q_{0}$ is constant, and zero in the right definite case. Suppose also that $p=w=1$ and $q=q_{0}$ a.e. in $(-\infty, a]$ for some $a \in \mathbb{R}$. Then the reflection coefficient $\mathfrak{R}_{-}$has a unique meromorphic extension to the upper half plane. For non-real $k^{2}$ we then have, in the right definite case that

$$
m\left(k^{2}\right)=i k \frac{1-e^{-2 i k a} \mathfrak{R}_{-}(k)}{1+e^{-2 i k a} \mathfrak{R}_{-}(k)},
$$

whereas in the left definite case we have

$$
m\left(q_{0}+k^{2}\right)=\frac{i k}{\lambda} \frac{1-e^{-2 i k a} \mathfrak{R}_{-}(k)}{1+e^{-2 i k a} \mathfrak{R}_{-}(k)} .
$$

In both cases $m$ denotes the corresponding Dirichlet $m$-coefficient for the interval $[a, \infty)$.

Pro of. In both cases we have $f_{-}(x, k)=e^{-i k x}$ for $x \leq a$, so that according to (3.4) we have

$$
\mathfrak{R}_{-}(k) / \mathfrak{T}(k)=\frac{e^{i k x}}{2 i k}\left(f_{+}(x, k) i k-p f_{+}^{\prime}(x, k)\right) .
$$

Thus $\mathfrak{R}_{-}$has a meromorphic extension to the upper half plane, with continuous boundary values on $\mathbb{R} \backslash\{0\}$ and poles precisely at the poles of $\mathfrak{T}$. According to Lemma 5.1 this extension is uniquely defined.

It follows that in $(-\infty, a]$ (3.2) is valid also in the upper half plane, away from the imaginary axis. If we also differentiate this formula we now have

$$
\begin{aligned}
& \mathfrak{T}(k) f_{+}(a, k)=\mathfrak{R}_{-}(k) e^{-i k a}+e^{i k a}, \\
& \mathfrak{T}(k) f_{+}^{\prime}(a, k)=-i k \mathfrak{R}_{-}(k) e^{-i k a}+i k e^{i k a} .
\end{aligned}
$$

Dividing the second formula by the first we obtain

$$
\frac{f_{+}^{\prime}(a, k)}{f_{+}(a, k)}=i k \frac{1-e^{-2 i k a} \Re_{-}(k)}{1+e^{-2 i k a} \Re_{-}(k)} .
$$

Noting, in the right definite case, that $p(a)=1$ we obtain the desired formulas, since $f_{+}$decays exponentially and therefore has finite norm over $[a, \infty)$ for $\operatorname{Im} k>0$.

The theorem shows that the reflection coefficient $\mathfrak{R}_{-}$determines the Dirichlet $m$-coefficient for $[a, \infty$ ) (and vice versa) if the equation has constant coefficients in $(-\infty, a]$. Together with Theorems 4.1, 4.4 and their corollaries we immediately obtain our main result.

Theorem 5.3 Under the assumptions of Theorems 5.2 and 4.1 in the right definite case and Theorem 4.4 in the left definite case the following is true.

(1) If in the right definite case $p$, or even just $|p|$, is known, then the potential $q$ is determined by $\mathfrak{R}_{-}$,

(2) If in the left definite case $w$, or even just $|w|$, and $q_{0}$ are known, then the potential $q$ is determined by $\mathfrak{R}_{-}$,

(3) If in the right definite case $q=0$, or in the left definite case $q$ and $q_{0}$ are known, then $p$, respectively $w$, is determined by $\Re_{-}$.

It should be noted that without a finite first moment for $q$ in the right definite case or for $q-q_{0}$ in the left definite case there may be infinitely many eigenvalues accumulating at 0 or $q_{0}$ respectively. Similarly, unless $p \geq 0$ in the right definite case and $w \geq 0$ in the left definite case there will definitely be infinitely many eigenvalues accumulating at $-\infty$ (see [6]).

Remark 5.4 If one does not know $q_{0}$ in the left definite case, then the $m$-coefficient is only determined up to a translation of the spectral parameter $\lambda$. This is natural, since we may also write the equation, e.g., as

$$
-f^{\prime \prime}+\left(q-q_{0} w\right) f=k^{2} w f .
$$


Clearly scattering data do not allow us to distinguish this equation from (2.3). One could alternatively formulate the left definite scattering problem as (2.3) where $w-1$ and $q$ is in $L^{1}(\mathbb{R})$ and there is a constant $q_{0}$ such that $q+q_{0} w \geq 0$ and not identically 0 . The appropriate norm-square is then the integral

$$
\|f\|^{2}=\int_{\mathbb{R}}\left(\left|f^{\prime}\right|^{2}+\left(q+q_{0} w\right)|f|^{2}\right) .
$$

The one-dimensional Schrödinger equation is the right definite equation (2.2) with $p=1$ and is therefore covered by Theorem 5.3(1).

The equation dealt with in acoustical scattering is of the form

$$
-f^{\prime \prime}=\lambda w f
$$

where $w>0$ and locally integrable. The change of variable $t=\int_{0}^{x} w$ will turn the equation into a right definite equation of the form (2.2) with $q=0$. This case is covered by Theorem 5.3(3).

The scattering problem associated with the Camassa-Holm equation is of the form

$$
-f^{\prime \prime}+\frac{1}{4} f=\lambda w f
$$

which is also covered by Theorem 5.3(3), now as a left definite problem.

It may seem surprising that no knowledge of the eigenvalues or the corresponding normalisation constants is required. However, the location of the eigenvalues is determined by the poles of the unique meromorphic extension of $\mathfrak{R}_{-}$to the upper half plane, and the normalisation constants by the corresponding residues, see also [1].

\section{Final comments}

It is clear that if the coefficients of (2.1) are instead constant in an interval $[a, \infty)$ one may similarly prove an analogue of Theorem 5.3 where the coefficients of the equation are instead determined from knowledge of $\mathfrak{R}_{+}$.

If the supports of $1-1 / p$ and $q$, respectively of $q-q_{0}$ and $w-1$, are not bounded in either direction it seems Titchmarsh-Weyl theory is of little use for inverse scattering. One may, however, create a spectral theory based on Jost solutions which is more relevant to scattering. It seems very general uniqueness theorems for inverse scattering may be deduced from this. We refer to [7].

Finally note that, if the coefficients $p, q$ and $w$ are known in $(-\infty, a]$ and such that $f_{-}$extends to an analytic function in the upper half plane, we can again express the Dirichlet $m$-function in terms of $f_{-}$and $\mathfrak{R}_{-}$, which also extends analytically to $\operatorname{Im} k>0$. We therefore obtain uniqueness theorems in the same way as before.

\section{References}

[1] T. Aktosun, M. Klaus, and C. van der Mee, On the Riemann-Hilbert problem for the one-dimensional Schrödinger equation, J. Math. Phys. 34(7), 2651-2690 (1993).

[2] C. Bennewitz, Spectral theory for pairs of differential operators, Ark. Mat. 15(1), 33-61 (1977).

[3] C. Bennewitz, Spectral asymptotics for Sturm-Liouville equations, Proc. Lond. Math. Soc. (3) 59(2), 294-338 (1989).

[4] C. Bennewitz, A Paley-Wiener theorem with applications to inverse spectral theory, in: Advances in Differential Equations and Mathematical Physics, Contemporary Mathematics Vol. 327 (Birmingham, AL, 2002, American Mathematical Society, Providence, RI, 2003), pp. 21-31.

[5] C. Bennewitz and B. M. Brown, A limit point criterion with applications to nonselfadjoint equations, J. Comput. Appl. Math. 148(1), 257-265 (2002), On the occasion of the 65th birthday of Professor Michael Eastham.

[6] C. Bennewitz, B. M. Brown, and R. Weikard, Inverse spectral and scattering theory for the half-line left-definite SturmLiouville problem, SIAM J. Math. Anal. 40(5), 2105-2131 (2009).

[7] C. Bennewitz, B. M. Brown, and R. Weikard, Scattering and inverse scattering for a left-definite sturm-liouville problem, Submitted, 2011.

[8] A. Constantin, On the scattering problem for the Camassa-Holm equation, R. Soc. Lond. Proc. Ser. A Math. Phys. Eng. Sci. 457(2008), 953-970 (2001).

[9] P. Deift and R. Killip, On the absolutely continuous spectrum of one-dimensional Schrödinger operators with square summable potentials, Commun. Math. Phys. 203(2), 341-347 (1999). 
[10] P. Deift and E. Trubowitz, Inverse scattering on the line, Commun. Pure Appl. Math. 32(2), 121-251 (1979).

[11] I. Egorova and G. Teschl, A Paley-Wiener theorem for periodic scattering with applications to the Korteweg-de Vries equation, Zh. Mat. Fiz. Anal. Geom. 6(1), 21-33, 134 (2010).

[12] L. D. Faddeev, Properties of the $S$-matrix of the one-dimensional Schrödinger equation, Tr. Mat. Inst. Steklova 73, 314-336 (1964).

[13] C.-E. Fröberg, Calculation of the interaction between two particles from the asymptotic phase, Phys. Rev. Minneapolis, II. Ser. 72, 519-520 (1947).

[14] F. Gesztesy and B. Simon, Inverse spectral analysis with partial information on the potential, I. The case of an a.c. component in the spectrum, Helv. Phys. Acta, 70(1-2), 66-71 (1997), Papers honouring the 60th birthday of Klaus Hepp and of Walter Hunziker, Part II (Zürich, 1995).

[15] B. Grébert and R. Weder, Reconstruction of a potential on the line that is a priori known on the half line, SIAM J. Appl. Math. 55(1), 242-254 (1995).

[16] E. Hille, Lectures on Ordinary Differential Equations (Addison-Wesley Publ. Co., Reading, Mass.-London-Don Mills, Ont., 1969).

[17] E. A. Hylleraas, Calculation of a perturbing central field of force from the elastic scattering phase shift, Phys. Rev. II. Ser. 74, 48-51 (1948).

[18] N. Levinson, On the uniqueness of the potential in a Schrödinger equation for a given asymptotic phase, Danske Vid. Selsk. Mat.-Fys. Medd. 25(9), 29 (1949).

[19] B. M. Levitan, Inverse Sturm-Liouville Problems, VSP, Zeist (1987), Translated from the Russian by O. Efimov.

[20] V. A. Marčenko, On reconstruction of the potential energy from phases of the scattered waves, Dokl. Akad. Nauk SSSR n. Ser. 104, 695-698 (1955).

[21] V. A. Marchenko, Sturm-Liouville Operators and Applications, Operator Theory Advances and Applications, Vol. 22 (Birkhäuser Verlag, Basel, 1986), Translated from the Russian by A. Iacob. 